\title{
LAS CADENAS DE SUPERMERADOS Y SU INCIDENCIA EN LA GENERACIÓN DE EMPLEO DEL CANTÓN JIPIJAPA
}

\author{
AUTORES: Jessenia Herminia Moran Chilan ${ }^{1}$ \\ Arturo Álvarez Indacochea ${ }^{2}$ \\ Maritza Sandra Pibaque Pionce ${ }^{3}$ \\ Jose Felix Peñafiel Loor ${ }^{4}$
}

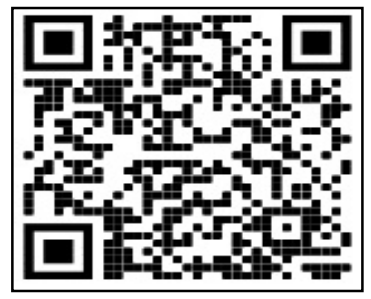

DIRECCIÓN PARA CORRESPONDENCIA: jesenia.moran@unesum.edu.ec

Fecha de recepción: 10/08/2020

Fecha de aceptación: 25/11/2020

\section{RESUMEN}

En este trabajo se propuso conocer de qué forma las cadenas de supermercados influyen en la generación de empleo del cantón Jipijapa, en donde los ciudadanos tengan la oportunidad de tener un empleo estable y seguro dentro de su mismo cantón. Para obtener la información se aplicaron diferentes métodos, tales como el método deductivo, inductivo y estadístico, los cuales se aplicaron para obtener información de las cadenas de supermercados constituidas en el cantón Jipijapa, las cuales son tres cadenas de supermercados que ofrecen 129 plazas de empleos para los ciudadanos del cantón. Las cadenas de supermercados se han posicionado fuertemente en el cantón de Jipijapa, lo cual genera fuentes de empleos para los ciudadanos, lo cual influye mucho en la calidad de vida de los ciudadanos. La investigación busca determinar la incidencia de estas cadenas en la generación de fuentes de trabajo, como también cuál es el porcentaje de plazas de trabajos generan estas cadenas de supermercados en el cantón, verificando si las plazas de trabajos son netamente para los ciudadanos del mismo cantón o también benefician a otros ciudadanos de cantones vecinos con ofertas de empleos. De acuerdo a lo desarrollado en la investigación es notable que los Supermercados del cantón de Jipijapa inciden positivamente en la economía; y sobre todo generando fuentes de trabajo por cuanto se toma en cuenta la mano de obra local.

PALABRAS CLAVE: Cadenas de supermercados, empleo, trabajo, sistema de contratación

\section{LAS CADENAS DE SUPERMERADOS Y SU INCIDENCIA EN LA GENERACIÓN DE EMPLEO DEL CANTÓN JIPIJAPA}

\section{ABSTRACT}

In this work, it was proposed to know how supermarket chains influence the generation of employment in the Jipijapa canton, where citizens have the opportunity to have a stable and

\footnotetext{
${ }^{1}$ Universidad Estatal del Sur de Manabí, Jipijapa, Ecuador. E-mail: jesenia.moran@unesum.edu.ec

${ }^{2}$ Universidad Estatal del Sur de Manabí, Jipijapa, Ecuador. E-mail: alvarezindacocheaarturoantonio@gmail.com

${ }^{3}$ Universidad Estatal del Sur de Manabí, Jipijapa, Ecuador. E-mail: $\underline{\text { m1_aritza@hotmail.com }}$

${ }^{4}$ Universidad Estatal del Sur de Manabí, Jipijapa, Ecuador. E-mail: jpfelix@hotmail.es
} 
Jessenia H. Moran Chilan, Arturo Álvarez Indacochea, Maritza S. Pibaque Pionce, Jose Felix Peñafiel Loor

secure job within the same canton. To obtain the information we apply different methods, such as the deductive, inductive and statistical method, which we apply to obtain information from the supermarket chains established in the Jipijapa canton, which are three supermarket chains that offer 129 jobs for the citizens of the canton Supermarket chains have strongly positioned themselves in the canton of Jipijapa, which generates sources of employment for citizens, which greatly influences the quality of life of citizens, the research seeks to determine the incidence of these chains in the generation of sources of work, as well as what is the percentage of jobs generated by these supermarket chains in the canton, verifying if the jobs are clearly for citizens of the same canton or also benefit other citizens of neighboring cantons with job offers. According to what has been developed in the research, it is notable that the supermarkets in our canton are contributing to the economy and above all generating sources of work since the local labor force is taken into account.

KEYWORDS: Supermarketchains, employment, work, hiringsystem

\section{INTRODUCCIÓN}

La implementación de las cadenas de supermercados en el ayudará al desarrollo socio económico del cantón, con todos sus beneficios sociales económicos y de sustentabilidad de desarrollo, al entrar en funcionamiento genera fuentes de empleos para las personas que residen en este cantón, además, es importante destacar la atención de calidad y calidez dada en una mejor atención al público, por consiguiente satisfacer las expectativas de los clientes brindando un servicio eficiente, con productos de primera necesidad las cuales se conservan en ambiente adecuado.

En el cantón Jipijapa la fuente de empleo es escasa ya que con la situación económica que atraviesa el país el cantón no genera fuente de empleo, sin embargo con la implementación de las cadenas de supermercados se genera en su mayor parte empleo a los habitantes del cantón Jipijapa. En virtud de lo señalado, se establece la importancia del presente estudio, el cual centra su atención en las cadenas de supermercado y su incidencia en la generación de empleo del cantón Jipijapa.

La evolución de los supermercados durante la década de los 90 ha evidenciando una expansión en varios sentidos, buscando una mayor competitividad. Para aumentar la rentabilidad, las redes de supermercados pasaron a vender también electrodomésticos, vestuarios y bienes de papelería. Hubo un incremento en las instalaciones a través de la elección de puntos bien ubicados para las tiendas, con establecimientos y otros servicios al consumidor.

En este trabajo de investigación se trata de un artículo de tipo analítico en la cual se comprenden la incidencia dentro de la generación de empleo en las cadenas de las distintas empresas o supermercados del cantón Jipijapa, a fin de enmarcar su aporte en el desarrollo local. En continuidad se resalta que el tipo de investigación a desarrollarse dentro de este artículo se utiliza lo siguiente tipos investigación bibliográfica, descriptiva, exploratoria y de campo.

\section{Investigación bibliográfica}

118 UNESUM-Ciencias. Publicación cuatrimestral. Vol. 4, No. 4 (Septiembre-Diciembre), Año 2020. 
Se utilizó este método de investigación bibliográfica ya que se extrajo información desde un inicio del estudio a través de la indagación de información en libros, revistas científicas, folletos e internet y otros medios electrónicos. Investigación exploratoria para poder conocer las distintas falencias dentro de los supermercados en las fuentes de empleo que generan para la sociedad la que se conlleva a cabo la investigación, mediante encuestas, fichas de observación y entrevistas. Investigación descriptiva para poder determinar con qué precisión se ha realizado esta investigación en las cadenas de supermercado y ver como inciden en la generación de empleo del cantón Jipijapa, y ver cómo se ha visto beneficiada la sociedad, mediante algunas técnicas como encuesta, entrevista y datos estadísticos. La Investigación campo se realizó en el cantón Jipijapa gracias a ella se pudo realizar la recopilación de datos de información a través de la técnica utilizada que fue de entrevista y el instrumento que fue el cuestionario de encuestas para luego representar en grafico de forma más visible y comprensible para las personas observantes

\section{DESARROLLO}

Un supermercado es aquel establecimiento que tiene como principal finalidad acercar los consumidores una importante variedad de productos de diversas marcas, precios y estilo. A diferencia de lo que sucede con gran parte de los negocios, un supermercado se caracteriza por exponer estos productos al alcance de los consumidores, quienes recurren al sistema de autoservicio y abonan la cantidad de ítems elegidos al final en la zona de cajas.

También busca sus objetivos, tantos generales como específicos, siendo ellos el pilar fundamental que aporta con el orden de ejecución como también la justificación donde se consideran desde diferentes puntos de vista la relevancia aportada, buscando resultados, la tabulación y el análisis de los datos obtenidos productos de las encuestas y se desarrolló las conclusiones que pudimos obtener mediante la información obtenida de las cadenas de supermercado situadas en el cantón Jipijapa. Planteando una propuesta.

En la actualidad los supermercados se encuentran en una constante búsqueda de alternativas para tratar de posesionar la imagen o marca en sus clientes. Uno de los factores claves y elementos diferenciador que otorga valor agregado a los supermercados es sin duda alguna la calidad del servicio a sus clientes. Abarcando desde el trato que se da al mismo, así como el aseo del lugar, el ambiente, el espacio entre los pasillos y el orden de los diferentes productos dentro de las perchas, entre otros.

Cadena de supermercado (Fisher \& Espejo, 2004) “en la práctica, cada persona tiene una definición de lo que es el mercado en función de lo que mejor le parece o le conviene, por ejemplo, para un accionista el mercado tiene relación con los valores o el capital; para una ama de casa, el mercado es el lugar donde compra los productos que necesita; desde el punto de vista de la economía, el mercado es el lugar donde se reúnen oferentes y demandantes y es donde se determinan los precios de los bienes y servicios a través del comportamiento de la oferta y la demanda."

La objetividad del consumidor, es decir el consumidor se abastece sus necesidades desde su punto de vista, dependiendo del capital que este tenga y de las necesidades que necesite suplir, 
Jessenia H. Moran Chilan, Arturo Álvarez Indacochea, Maritza S. Pibaque Pionce, Jose Felix Peñafiel Loor

dependiendo también de la economía en la que se encuentre. Ya que el mercado oferta producto de diferentes valores y marcas y muchas veces el consumidor no abastece de tantos recursos económicos para adquirir dicho producto, lo que produce una búsqueda de un producto más barato o buscar un suplemento.

(Stanton, Etzel, \& Walker, 2004), definen el mercado como "las personas u organizaciones con necesidades que satisfacer, dinero para gastar y voluntad de gastarlo”. El mercado se conforma por el vendedor y el comprador en donde el vendedor es el que oferta los productos al mercado, es decir, a los consumidores, en donde ellos se encargaran de conseguir los recursos económicos para poder así obtener estos productos para poder satisfacer las necesidades que tengan.

El mercado es "donde confluyen la oferta y la demanda. En un sentido menos amplio, el mercado es el conjunto de todos los compradores reales y potenciales de un producto. Por ejemplo: El mercado de los autos está formado no solamente por aquellos que poseen un automóvil sino también por quienes estarían dispuestos a comprarlo y disponen de los medios para pagar su precio" (Bonita \& Farber, 2004).

El mercado es un lugar en donde mucha gente concurren a obtener productos para satisfacer sus necesidades, estos productos lo pueden conseguir con dinero, ya que actualmente el único recurso para obtener algún bien es el dinero y tener la voluntad de poder gastarlo en cualquier bien o servicio que creamos que sea necesario para nuestra vida. Además el mercado es un amplio segmento donde se incluyen comerciantes, vendedores, productores, empresas intermediarios entre otros.

Para los autores (Philip, Gary, Dionisio, \& Roche, 2004) un mercado es el "conjunto de compradores reales y potenciales de un producto. Estos compradores comparten una necesidad o un deseo particular que puede satisfacerse mediante una relación de intercambio". Las necesidades de los consumidores son muy importantes para el mercado, ya que de ellos saldrá el tipo de ofertas que estarán en el mercado, existen muchos productos y servicio que se encuentran en un mercado, todos con el objetivo de satisfacer las necesidades de los consumidores, muchos adecuándose a la economía de ellos.

Las empresas entienden que satisfacer las necesidades es dar, entregar un producto que realmente satisfaga a cada uno de los clientes, que están diferenciados por edades, sexo, clases, culturas e idiosincrasia proveniente del núcleo familiar, para ello estas empresas emplean un sinnúmero de marketing que ayude a posesionarse en el mercado.

Mientras, para (Mankiw, 2002) un mercado es "un grupo de compradores y vendedores de un determinado bien o servicio. Los compradores determinan conjuntamente la demanda del producto, y los vendedores, la oferta". Los consumidores son la demanda de la oferta, este visualizara y probara el producto para ver su calidad y saber si es el indicado para satisfacer las necesidades, los productos con más demanda en el mercado, son los productos que sirvan para las necesidades básicas, ya que todos los consumidores las tenemos y las necesitamos comprar, y los ofertantes son los encargados de llevar estos productos al mercado, siendo de diferentes marcas y precios, pero con el mismo fin, que es el de satisfacer y suplir las necesidades de los consumidores lo mejor posible.

120 UNESUM-Ciencias. Publicación cuatrimestral. Vol. 4, No. 4 (Septiembre-Diciembre), Año 2020. 
(Rosario, 2019) dice que "Clarence Saunders fue un pionero creando el primer supermercado del mundo en el que se le ocurrió poner a merced de los compradores las mercancías para su venta. Su objetivo era ahorrar tiempo y trabajo para el vendedor, y lo consiguió. De esa forma nació en 1916 PigglyWiggly, la primera cadena de supermercados del mundo en la que el cliente podía comprar y observar los productos y pagar a la salida."

El intercambio de productos para la satisfacción de las necesidades se lleva a cabo desde los principios del ser humano, pero la idea de unir todos los productos en un solo lugar, fue la cadena de supermercado "PigglyWiggly" en donde los consumidores podían ir y elegir entre todos los productos y poder obtener su producto en un mismo almacén.

(Tapia, 2015) plantea que en "Ecuador el total de la producción nacional se encuentra dividida entre 16 sectores industriales: industrias manufactureras; comercio al por mayor y al por menor; explotación de minas y canteras; agricultura, ganadería, silvicultura y pesca, transporte, almacenamiento y comunicaciones; construcción; administración pública y defensa, planes de seguridad social de afiliación obligatoria; enseñanza; actividades profesionales, técnicas y administrativas; servicios sociales y de salud; intermediación financiera; hoteles y restaurantes; suministro de electricidad y agua; hogares privados con servicio doméstico; otros servicios; y otros elementos del PIB.”

El mismo indica que: Dentro del entorno de los supermercados en Ecuador se distingue la existencia de diferentes nichos de mercados, los cuales se diferencian principalmente por la capacidad adquisitiva de la población. el nivel socioeconómico de la población ecuatoriana se encuentra divido en 5 categorías.la primera categoría, en la que se encuentra tan solo el 1,9\% de la población y con un mayor poder adquisitivo es la categoría A; la segunda categoría es la B con el 11,2\% de la población ecuatoriana, en tercer y cuarto lugar están las categorías C+ y C- que agrupan al $72,10 \%$ de la población y finalmente el nivel final (D) que tiene al 14,9\% de la población y agrupa a las personas con los menores ingresos respecto a la población total.

De igual manera expresa que "Corporación La Favorita posee cuatro grandes marcas de supermercados: la primera, Supermaxi y Megamaxi, cuyos productos principalmente se enfocan en la población que se encuentra en el nivel socioeconómico A, B e incluso C+, mientras que las marcas Aki y Gran Aki se enfocan en la comercialización de productos hacia el porcentaje de población que se encuentra principalmente en el nivel socioeconómico C+ y C-. De esta manera, Corporación La Favorita puede comercializar con más de un nivel socioeconómico y beneficiarse de la demanda de cada uno de ellos.

En Ecuador existen muchas las cadenas se supermercado de diferentes tipos que se pueden encontrar, en las cuales se encontrar cualquier variedad de productos, desde comida, hasta ferretería.

El trabajo es definido según (TORRES, 2005) como: "Es el esfuerzo humano, físico o intelectual, aplicado a la producción u obtención de la riqueza. Toda actividad susceptible de valoración económica por la tarea, el tiempo o el rendimiento. Ocupación de conveniencia social o individual, dentro de la licitud”. 
Jessenia H. Moran Chilan, Arturo Álvarez Indacochea, Maritza S. Pibaque Pionce, Jose Felix Peñafiel Loor

El trabajo es un derecho y un deber social para las personas, en la forma y con las limitaciones prescritas en la Constitución y las leyes ecuatorianas. El trabajador es libre para dedicar su esfuerzo a la labor lícita que a bien tenga. Ninguna persona podrá ser obligada a realizar trabajos gratuitos, ni remunerados que no sean impuestos por la ley, salvo los casos de urgencia extraordinaria o de necesidad de inmediato auxilio. Fuera de esos casos, nadie estará obligado a trabajar sino mediante un contrato y la remuneración correspondiente. En general, todo trabajo debe ser remunerado.

Desde el punto de vista lógica, el trabajo es un derecho y un deber para las personas en lo comprende o se denomina a la realización de todo tipo de acción realizada por el talento y capacidad de una persona en dicha actividad laboral por lo cual deberá ser muy bien compensado o remunerado, que por lo general tiene que ver con la obtención de metas y la satisfacción de necesidades en beneficio económico de la persona que realiza el trabajo, así como lo regula el código de trabajo.

\section{Sistema de contratación}

La doctrina laboral ha emitido diferentes criterios acerca de la clasificación de los contratos de trabajo en relación de dependencia; con lo que coinciden varios tratadistas es que en algunos casos existen semejanzas con respecto a nuestro Código de Trabajo. Para este estudio y en base a las disposiciones emitidas por la ley, se han adoptado cinco criterios por los que se distinguen, estos son:

1. Expreso o tácito, y el primero, escrito o verbal

2. A sueldo, a jornal, en participación y mixto

3. Por tiempo fijo, por tiempo indefinido, de temporada, eventual y ocasional

4. A prueba

5. Por obra cierta, por tarea y a destajo

6. Por enganche

7. Individual, de grupo o por equipo

Contratos expreso y tácito: El contrato es expreso cuando el empleador y el trabajador acuerden las condiciones, sea de palabra o reduciéndolas a escrito.

Contratos a sueldo y a jornal: la remuneración se pacta tomando como base, cierta unidad de tiempo. Contrato en participación es aquel en el que el trabajador tiene parte en las utilidades de los negocios del empleador, como remuneración de su trabajo. La remuneración es mixta cuando, además del sueldo o salario fijo, el trabajador participa en el producto del negocio del empleador, en concepto de retribución por su trabajo

Por tiempo fijo: Establéese un tiempo mínimo de duración, de todo contrato por tiempo fijo o por tiempo indefinido, que celebren los trabajadores con empresas o empleadores en general, cuando la actividad o labor sea de naturaleza estable o permanente, sin que por esta circunstancia los contratos por tiempo indefinido se transformen en contratos a plazo, debiendo considerarse a tales trabajadores para los efectos de esta Ley como estables o permanentes. 
Contrato a prueba: son aquellos a los que se refiere el inciso primero del artículo anterior, cuando se celebre por primera vez, podrá señalarse un tiempo mínimo de prueba, de duración máxima de noventa días.

Contratos por obra cierta, por tarea y a destajo: El contrato es por obra cierta, cuando el trabajador toma a su cargo la ejecución de una labor determinada por una remuneración que comprende la totalidad de la misma, sin tomar en consideración el tiempo que se invierta en ejecutarla.

Contratos eventuales, ocasionales, de temporada: Son aquellos que se realizan para satisfacer exigencias circunstanciales del empleador, tales como reemplazo de personal que se encuentra ausente por vacaciones, licencia, enfermedad, maternidad y situaciones similares. (Código del trabajo)

En resumen, de lo dispuesto en código de trabajo en regulación de los deberes y derechos que tiene un trabajador dependiendo del tipo de servicio que realice tanto físico como intelectual estos comprenden según al distinto sistema de contratación a los que se ajusten según su profesión. Además, este código de trabajo nos dice que los deberes y derechos de los son irrenunciables.

En este contexto, en (Caraballo, 2017) se plantea:

"En los estudios realizados por las ciencias sociales acerca del trabajo se encuentra una diversidad de enfoques, metodologías y niveles de análisis desde diversas perspectivas como la económica, histórica, social y psicológica. La evolución del constructo ha ido desde la distinción entre labor y trabajo hasta considerarla como actividad degradante, castigadora o, por el contrario, dignificante, ubicándose en un espacio central en la vida de los individuos. Este artículo de revisión aborda el fenómeno del trabajo desde una perspectiva histórica, ubicando definiciones y la configuración del concepto, desde la época grecorromana hasta la década de los años setenta del siglo XX. Se recalcan condiciones constitutivas de la configuración de significado como son la escala de valores, las circunstancias, el contexto y la socialización con el trabajo. Por qué se trabaja y la importancia de trabajar hacen parte de la configuración del significado del trabajo presentada en esta revisión.”

El trabajo del ser humano se viene dando desde tiempos neolíticos, ya que todas las personas para poder obtener un valor adquisitivo de ingreso, tienen que trabajar, realizar sus labores de trabajo en cualquier cosa que genere dinero, el trabajo hace mucho tiempo se creía que era una forma de castigar, de resignación por algún castigo, pero con el tiempo el trabajo fue convirtiéndose como una fuente de generación de dinero para poder así sobrevivir.

Para los autores (Pérez Porto \& Merino, 2012), "la definición teórica del concepto de trabajo puede ser analizada desde perspectivas distintas. Una aproximación básica presenta a este vocablo como la valoración del esfuerzo realizado por un ser humano. Sin embargo, desde la perspectiva neoclásica de la economía, por citar otro caso a modo de ejemplo, el trabajo consiste en uno de los tres elementos que determinan toda producción, como sucede con la tierra y el 
Jessenia H. Moran Chilan, Arturo Álvarez Indacochea, Maritza S. Pibaque Pionce, Jose Felix Peñafiel Loor

dinero. De esta manera, y aproximándonos a lo que de manera habitual todos los ciudadanos entendemos por trabajo, podemos determinar que dicho concepto se puede utilizar de dos maneras muy frecuentes. Así, por un lado, nos referimos a él como la acción que realiza una persona que se está ocupando de llevar a cabo una serie de tareas o actividades ya sea a nivel físico o bien intelectual y por otro lado, utilizamos este mismo término para hacer referencia a toda ocupación que tiene un hombre o una mujer fuera de su hogar por la cual recibe una asignación económica mensual.”

Además expresa que no obstante, además de todo lo expuesto hay que subrayar que trabajo es un sustantivo que cuenta con otras muchas más acepciones. De esta forma también se emplea para hacer referencia a lo que es el lugar en sí donde se lleva a cabo el desarrollo de la ocupación. Y todo ello sin olvidar tampoco que existe una serie de expresiones que utilizan el concepto que nos ocupa como parte fundamental. Así, también se habla de trabajos forzados que son aquellos que realizan de manera obligada y como parte de su condena las personas que se encuentran en ciertas prisiones.

"Deriva del término latino, "tripalium", que significa tres palos, que se empleaban como instrumento de tortura. El castellano arcaico cambió el vocablo a "trebejare”, y de allí pasó a nuestro idioma como trabajo. Es toda actividad humana lícita, remunerada, que expresa la capacidad creativa del hombre, manifestada como esfuerzo físico, intelectual o artístico. Extensivamente puede usarse el término para designar la acción de la herramienta, utensilio, o máquina que la persona utiliza para realizar su propio trabajo personal. En ocasiones se usa el término para designar la actividad creativa de otros seres de la naturaleza, como cuando se dice: “las abejas, trabajan, elaborando la miel”. La diferencia sustancial entre este trabajo y el del hombre, es que los otros seres vivos, realizan un esfuerzo mecánico, y no remunerado, para obtener un fin, que es ajeno a su conciencia. El fin del trabajo humano, en última instancia, hace a su dignidad como persona.”(DeConceptos.com, 2019)

El Trabajo como "todo tipo de acción realizada por el hombre independientemente de sus características o circunstancias; significa toda la actividad humana que se puede o se debe reconocer como trabajo entre las múltiples actividades de las que el hombre es capaz y a las que está predispuesto por la naturaleza misma en virtud de su humanidad. La necesidad de trabajar quizás tuvo su origen, hace milenios, en el instinto básico del hombre de sobrevivir y perpetuarse como especie. En aquel mundo solo y hostil, el hombre debió utilizar todas sus potencialidades para proveerse de alimentos, elaborar su ropa y vivienda, fabricar sus utensilios, herramientas y armas, para proteger a sus hijos.”

\section{RESULTADOS}

En los supermercados constituidos en la ciudad de Jipijapa tenemos que los empleados que realizan sus labores diarias por contratos establecidos que son amparados por la ley, que un $48 \%$ comprende entre la edad de 25 a 30 años, mientras que un 32\% esta entra las edades de 20 a 25 años, de 18 años a 20 años solo se encuentra el 2\% de trabajadores y con un 19\% tienen desde 35 años en adelante. 
En relación al análisis de género realizado, los trabajadores que realizan sus labores diarias en las cadenas de supermercados del cantón Jipijapa. El 54\% son de género femenino, mientras que el $46 \%$ son de género masculino, es decir que las ofertas laborales de las cadenas de supermercado, si hay un equilibrio por género.

En relación a tributos para la ciudad Los trabajadores encuestados, solo el $67 \%$ cree que las cadenas de supermercados constituidas en la ciudad de Jipijapa generan ingresos económicos a la ciudad, por medio de impuestos pagados, mientras que el 33\% expreso que lo hace mediante otros métodos, mientras que nadie cree que las cadenas de supermercados cancelan tributos para el desarrollo de la ciudad.

Las Cadenas de supermercados constituidas en la ciudad de Jipijapa, Genera empleos para los habitantes de la ciudad, pero solo genera dos únicas vacantes, las cuales son Cajero y Percheros, donde el $47 \%$ de trabajadores encuestados, expresaron que sus labores diarias las hacen en cajero, mientras que el 53\% obtuvieron vacantes para realizar sus labores como percheros.

En relación a capacitaciones profesionales, solamente el 72\% están capacitados para ejercer sus labores dentro de la organización, ya que expresaron mediante las encuestas que, si realizaron capacitaciones para el conocimiento del área donde realizan sus labores de trabajo, mientras que solo el 6\% no están capacitados, ya sean por que recién entraron a trabajar, ya que la compañía si genera capacitaciones para sus trabajadores.

En la ciudad de Jipijapa, se encuentran constituidas tres importantes cadenas de supermercados del país, las cuales son Mi comisariato, Tía, Akí. De las cuales por medio de las encuestas dijeron que el $39 \%$ de los trabajadores, realizan sus labores de trabajo en mi comisariato el $36 \%$ son trabajadores pertenecientes a la compañía Tía y el 25\% son de la cadena de supermercados Akí.

Los trabajadores de estas cadenas de supermercados firman un contratos de los cuales muchos son contratos a prueba, mediante las encuestas realizadas a los trabajadores de estas cadenas de supermercados, podemos decir que el $74 \%$ de los trabajadores, es decir la mayoría de trabajadores son cumplen sus labores de trabajo por un contrato a prueba, es decir por un contrato temporal de 3 meses, el $2 \%$ tienen un contrato ocasional, el 19\% tienen contrato rotativo y solo el $4 \%$ tienen un contrato profesional.

Las encuestas realizadas, fueron a 129 trabajadores de distintas cadenas de supermercados de la ciudad de Jipijapa, en donde el 100\% si está de acuerdo al horario establecido por estas cadenas de supermercados, pero estos trabajadores quieren también comenzar con sus estudios académicos, en donde propusimos propuestas si se llegara a tener la oportunidad de que la empresa de permiso para empezar los estudios, como recompensarían estas horas, de los cuales el 51\% de los trabajadores, es decir la mitad de trabajadores encuestados dijeron que recuperarían estas horas los sábados y domingos, mientras que el 30\% hicieran horas extras, y solo el 19\% expreso que sacrificarían los feriados para recompensar las horas perdidas por la oportunidad de empezar los estudios académicos. 
Jessenia H. Moran Chilan, Arturo Álvarez Indacochea, Maritza S. Pibaque Pionce, Jose Felix Peñafiel Loor

De las encuestas realizadas a la población del cantón Jipijapa, provincia de Manabí en donde se pudo observar que de todas las personas encuestadas con referente a los datos obtenidos y a su vez permitiendo llegar a los siguientes resultados, se deja establecido que las cadenas de supermercado dentro del cantón Jipijapa generan empleo a los habitantes del mismo, ya que así estas entidades o cadenas de supermercados puedan fomentar la actividad económica y el desarrollo local de dicho cantón.

Las cadenas de supermercado son establecimientos de ventas al detalle con muchas secciones, que vende comestibles y algunas u otras mercancías en beneficio y satisfacción de alguna necesidad para la población, de propiedad total del que lo lleva a lo bien funcionando por concesión, con un espacio adecuado para el establecimiento en la generación de empleo mediante estas cadenas de supermercado dentro del cantón.

Con los referentes a los datos obtenidos permitieron a llegar a los siguientes resultados, se deja establecido que las cadenas de supermercado en el cantón Jipijapa generan empleo a los habitantes del cantón, ya que así estas puedan fomentar la actividad económica y el desarrollo local de dicho cantón.

De acuerdo a las encuestas y entrevista realizada a los empleados y administradores de las cadenas de supermercados del cantón jipijapa se pudo evidenciar otros resultados.

\section{RESULTADOS}

La presencia de las cadenas de supermercados en el cantón jipijapa, generan desarrollo económico al cantón lo cual lo demostramos con la siguiente información, tal es el caso de la Corporación el Rosado - Mi Comisariato S.A/ Mi Comisariato jipijapa, donde se describe la actividad principal como "ventas al por menor de artículos de ferreterías, ventas al por menor de motocicletas y trineos motorizados, ventas al por menor de juguetes, ventas al por menor de prendas de vestir, ventas al por menor de gran variedad de productos de supermercado

Dentro de las cadenas de supermercados la mayoría de los empleados/as encuestados posee un $48 \%$, con una edad de 25 a 30 años, seguido del 32\% con la edad de 20 a 25 años, siendo también la mayor parte que laboran en estas cadenas o establecimientos del cantón jipijapa. Además, se evidencia que la mayoría de los empleados/as pertenece al género femenino con un 54\%, mientras que el género masculino con un $46 \%$, lo cual la mayoría de personas que laboran en los supermercados son de género femeninas.

Es importante también conocer que estas empresas generan el desarrollo económico dentro de la ciudad. En donde se conoció que el 67\% de estas empresas generan impuestos a la cuidad, seguido de un 33\% de manera que también genera desarrollo económico a la cuidad de Jipijapa, mientras tanto que los empleados/as cumplen funciones laborales dentro de las cadenas de supermercados. Por consiguiente, dentro de los datos recolectados se percibe que un $53 \%$ en funciones laborales en los empleados son percheros seguido de $47 \%$ que son cajeros, lo cual se determina que la mayoría del personal que laboran en estos supermercados son percheros, es muy importante recalcar que el personal si están capacitados para ejercer dicho cargo dentro de las cadenas de supermercados. 
A continuación, se muestran que el 72\% de los empleados si están capacitados para ejercer dichas funciones dentro de los establecimientos de los supermercados, seguido con un $28 \%$ de los empleados que no están capacitados, en conclusión, que la mayor parte del personal pueden ejercer funciones en estos supermercados tanto como en Tía, Aki u otras empresas ya sea mediana y pequeñas microempresas de algún emprendimiento.

La corporación favorita AKI jipijapa, también aporta con la economía a la ciudad, destacando el pago de impuesto patente donde se describe la actividad principal como "ventas al por menor de diversos productos. Ventas al por menor de alimentos, bebidas y tabaco en supermercados. I según oficio na0428-csepm-noc-2019-de fecha mayo 10/2019-emitido por el departamento de comisaría municipal, en el cual se cancela un valor de $\$ 789.30$ dólares americanos, de igual forma se aporta por impuesto del 1,5xmil sobre los activos totales de conformidad la ordenanza en vigencia un valor de $\$ 4077.11$ dólares americanos.

De acuerdo a lo desarrollado en la investigación es notable que los Supermercados en nuestro cantón están aportando a la economía y sobre todo generando fuentes de trabajo por cuanto se toma en cuenta la mano de obra local. Los habitantes del cantón Jipijapa se ha beneficiado con la presencia de los Supermercados en el medio, ya que de esta manera directa aproximadamente 129 empleados forman parte de estas cadenas de supermercados en el cantón.

La mano de obra de los ciudadanos Jipijapenses han sido tomados en cuenta para que formen parte del equipo de trabajo o empleados de estas entidades comerciales, es importante resaltar que los trabajadores de dichos establecimientos en un principio son contratados por un periodo de tres meses, pasado este tiempo y considerando el desempeño del trabajador se extiende el contrato hasta que la empresa lo determine según las funciones y responsabilidades que este posee. Es destacable indicar que las familias de los empleados han mejorado sus estatus de vida, debido a que la permanencia en el trabajo beneficia a los empleados, al poseer alimentación, salud y educación constante.

Indirectamente las personas que se han beneficiado son las personas que reciclan que están pendiente del material que se recicla como lo es fundas, cartón y plásticos que desechan las cadenas de supermercado, las personas que también se benefician son los taxistas que se han beneficiado con los movimientos que generan estos supermercados.

Al momento las cadenas de Supermercados, utilizan el sistema de contratación FIJA con sus empleados considerando los derechos que todo trabajador debe de tener, contratación que estipula las 40 horas semanales de trabajo y 2 días de descanso. Esto permite que los trabajadores cumplan con trabajo eficiente, brindando servicios de calidad y calidez a todos y cada uno de los consumidores que visitan los diferentes Supermercados.

\section{REFERENCIAS BIBLIOGRÁFICAS}

Bonita, P., \& Farber, M. (2004). 199 Preguntas sobre Marketing y Publicidad. Barcelona: Norma.

Caraballo, M. P. (2017). Significado del trabajo desde la psicología del trabajo. Una revisión histórica, psicológica y social. Scielo, 34. 
Jessenia H. Moran Chilan, Arturo Álvarez Indacochea, Maritza S. Pibaque Pionce, Jose Felix Peñafiel Loor

Código del trabajo. (2013). Corporación de Estudios y publicaciones. Ecuador: CDT.

Conceptodefinicion.de. (17 de Julio de 2019). Definición de Trabajo. Obtenido de https://conceptodefinicion.de/trabajo/

DeConceptos.com. (2019). Concepto de Trabajo. Obtenido de https://deconceptos.com/ciencias-naturales/trabajo

Fisher, L., \& Espejo, J. (2004). Mercadotecnia (Tercera Edición ed.). Mexico: McGraw-Hill.

Mankiw, G. N. (2002). Principios de Economía. España: McGraw-Hill.

Pérez Porto, J., \& Merino, M. (2012). Definición.de. Obtenido de Definición de trabajo: https://definicion.de/trabajo/

Philip, K., Gary, A., Dionisio, C. I., \& Roche, I. C. (2004). Marketing (Décima Edición ed.). Madrid, España: Pearson-Prentice Hall.

Rosario, P. (2019). Economipedia. Obtenido de Supermercado: https://economipedia.com/definiciones/supermercado.html

Stanton, W. J., Etzel, M. J., \& Walker, B. J. (2004). Fundamentos de Marketing (13 Edición ed.). Mexico: McGrawHill Interamericana.

Tapia, C. O. (2015). Supermercado en Ecuador: oligopolios e implicaciones de la Ley Orgánica de Rehulación y Control del Poder de Mercado. Quito: PUCE. Obtenido de http://repositorio.puce.edu.ec/bitstream/handle/22000/8393/Disertaci\%C3\%B3n\%20Carolina\%20Obando\%20 Tapia.pdf?sequence $=1$ \&isAllowed $=\mathrm{y}$

TORRES, C. D. (2005). Definición del trabajo (Pág. 376 ed.). México.

128 UNESUM-Ciencias. Publicación cuatrimestral. Vol. 4, No. 4 (Septiembre-Diciembre), Año 2020. 